\title{
Differential and difference polynomial sequences
}

\author{
Veasna Kim $^{1}$, Vichian Laohakosol ${ }^{2}$ \\ and Supawadee Prugsapitak ${ }^{1}$ \\ ${ }^{1}$ Department of Mathematics and Statistics, Faculty of Science \\ Prince of Songkla University, Hat Yai 90110, Thailand \\ e-mails: kim.veasna909@gmail.com, supawadee.p@psu.ac.th \\ ${ }^{2}$ Department of Mathematics, Faculty of Science \\ Kasetsart University, Bangkok 10900, Thailand \\ e-mail: fscivileku.ac.th
}

Received: 10 July 2019

Revised: 15 October 2019

Accepted: 21 October 2019

\begin{abstract}
A polynomial sequence is a sequence of $n$ positive integers which represents the values of an integer polynomial at the first $n$ positive integers. We extend this notion to differential and difference polynomial sequences which are defined analogously by incorporating not only the polynomial values but also the values of its derivatives and/or differences at integer points. Characterizations and their algebraic structures are determined.
\end{abstract}

Keywords: Polynomial sequence, Differential polynomial sequence, Difference polynomial sequence.

2010 Mathematics Subject Classification: 11B83, 11C08, $13 \mathrm{G} 05$.

\section{Introduction}

Throughout the entire paper, $n$ is a fixed positive integer. By a polynomial sequence (of length $n$ ), we mean a sequence $\mathbf{a}:=\left(a_{1}, a_{2}, \ldots, a_{n}\right) \in \mathbb{Z}^{n}$ for which there exists $f(x) \in \mathbb{Z}[x]$ such that $f(i)=a_{i} \quad(i=1,2, \ldots, n)$, and $f(x)$ is referred to as a polynomial which generates the sequence a. Denote by $P_{n}$ the set of all polynomial sequences. Cornelius and Schultz in [1] characterized $P_{n}$ using Lagrange and (implicitly) Newton interpolation polynomials and determined the structure of $\mathbb{Z}^{n} / P_{n}$. In [4], the results of Cornelius-Schultz have been generalized from $\mathbb{Z}$ to an integral domain $D$. 
Our first objective here is to extend these results further to differential polynomial sequences, the concept that we now describe. Let $I=\left(i_{1}, i_{2}, \ldots, i_{n}\right) \in D^{n}$ with $i_{s} \neq i_{t}$ for $s \neq t$, let $r_{j} \in \mathbb{N}_{0}:=\mathbb{N} \cup\{0\} \quad(j=1, \ldots, n)$, and let

$$
A=\left(a_{1}^{(0)}, a_{1}^{(1)}, \ldots, a_{1}^{\left(r_{1}\right)}, a_{2}^{(0)}, a_{2}^{(1)}, \ldots, a_{2}^{\left(r_{2}\right)}, \ldots, a_{n}^{(0)}, a_{n}^{(1)}, \ldots, a_{n}^{\left(r_{n}\right)}\right) \in D^{r_{1}+\cdots+r_{n}+n} .
$$

If there exists $f(x) \in D[x] \backslash\{0\}$ such that

$$
f^{(m)}\left(i_{j}\right)=a_{j}^{(m)} \quad\left(m=0,1, \ldots, r_{j} ; j=1,2, \ldots, n\right),
$$

then $A$ is referred to as a D-pol seq (differential polynomial sequence) of order $R=\left(r_{1}, \ldots, r_{n}\right)$ with respect to $I$, and denote the set of all differential polynomial sequences by $\wp(R, I)$. It is easy to check that the set $\wp(R, I)$ is an abelian group under addition, and for $c \in D$ if $A \in \wp(R, I)$, then $c \cdot A \in \wp(R, I)$, which shows that $\wp(R, I)$ is a $D$-module.

Our second objective is to introduce and investigate the concept of difference polynomial sequences. Let $I=(1,2, \ldots, n) \in \mathbb{Z}^{n}$. For a polynomial $f(x)$, define its differences, [2, Section 2.7], by

$$
\begin{aligned}
(\Delta f)(x) & =\left(\Delta^{1} f\right)(x):=f(x+1)-f(x) \\
\left(\Delta^{n} f\right)(x) & :=\left(\Delta^{n-1} f\right)(x+1)-\left(\Delta^{n-1} f\right)(x) \quad(n \geq 2) .
\end{aligned}
$$

For $k \in\{1,2, \ldots, n-1\}$, let

$$
\begin{aligned}
& \mathbb{Z}[x]_{n}:=\{f(x) \in \mathbb{Z}[x]: \operatorname{deg} f<n\} \\
& \Delta^{k} \mathbb{Z}[x]_{n}:=\left\{g(x) \in \mathbb{Z}[x]_{n-k}: \text { there exists } f(x) \in \mathbb{Z}[x]_{n} \text { such that }\left(\Delta^{k} f\right)(x)=g(x)\right\} \\
& \Delta^{k} P_{n}:=\left\{\mathbf{b}:=\left(b_{1}, \ldots, b_{n-k}\right) \in \mathbb{Z}^{n-k}: \text { there is } f(x) \in \mathbb{Z}[x] \text { satisfying }\left(\Delta^{k} f\right)(i)=b_{i}\right. \\
& (1 \leq i \leq n-k)\}
\end{aligned}
$$

The set $\Delta^{k} P_{n}$ is referred to as a $\Delta^{k}$-pol seq ( $k^{\text {th }}$ difference polynomial sequence). The last part of this work is to derive characterizations and related direct sum decompositions of the set $\Delta^{k} P_{n}$.

\section{Differential polynomial sequences}

Recall, [5, Theorem 1], that there is a unique polynomial

$$
H(x):=H_{A, I}(x) \in D_{Q}[x] \quad\left(D_{Q} \text { the quotient field of } D\right)
$$

of degree less than $n+\sum_{j=1}^{n} r_{j}$ satisfying the same relations as in (1), viz.,

$$
H^{(m)}\left(i_{j}\right)=a_{j}^{(m)} \quad\left(0 \leq m \leq r_{j}, 1 \leq j \leq n\right) .
$$

This polynomial, referred to as a generalized Hermite interpolation polynomial, has the following explicit form

$$
H(x)=\sum_{j=1}^{n} \sum_{m=0}^{r_{j}} A_{j, m}(x) a_{j}^{(m)},
$$


where

$$
\begin{aligned}
A_{j, m}(x) & =L_{j}(x) \frac{\left(x-i_{j}\right)^{m}}{m !} \sum_{t=0}^{r_{j}-m} \frac{1}{t !} g_{j}^{(t)}\left(i_{j}\right)\left(x-i_{j}\right)^{t} \\
L_{j}(x) & =\left(x-i_{1}\right)^{r_{1}+1}\left(x-i_{2}\right)^{r_{2}+1} \cdots\left(x-i_{j-1}\right)^{r_{j-1}+1}\left(x-i_{j+1}\right)^{r_{j+1}+1} \cdots\left(x-i_{n}\right)^{r_{n}+1} \\
g_{j}(x) & =1 / L_{j}(x) .
\end{aligned}
$$

We start with a characterization of $\wp(R, I)$.

Theorem 2.1. Keeping the above notation, the sequence

$$
A=\left(a_{1}^{(0)}, a_{1}^{(1)}, \ldots, a_{1}^{\left(r_{1}\right)}, a_{2}^{(0)}, a_{2}^{(1)}, \ldots, a_{2}^{\left(r_{2}\right)}, \ldots, a_{n}^{(0)}, a_{n}^{(1)}, \ldots, a_{n}^{\left(r_{n}\right)}\right)
$$

is a D-pol seq (of order $R$ with respect to $I$ ) if and only if its generalized Hermite interpolation polynomial $H(x)$, as in (2), is in $D[x]$.

Proof. If $A \in \wp(R, I)$, then there exists $f(x) \in D[x]$ such that (1) holds. Let

$$
p(x):=\left(x-i_{1}\right)^{r_{1}+1}\left(x-i_{2}\right)^{r_{2}+1} \cdots\left(x-i_{n}\right)^{r_{n}+1} \in D[x], \operatorname{deg} p(x)=n+\sum_{j=1}^{n} r_{j} .
$$

Since $p(x)$ is monic, by the division algorithm, $f(x)=q(x) p(x)+r(x)$, where $q, r \in D[x]$ with $r \equiv 0$ or $\operatorname{deg} r<n+\sum_{j=1}^{n} r_{j}$. Taking derivatives, we get

$$
f^{(k)}(x)=\sum_{m=0}^{k}\left(\begin{array}{c}
k \\
m
\end{array}\right) p^{(m)}(x) q^{(k-m)}(x)+r^{(k)}(x) \quad\left(1 \leq k \leq r_{j}\right) .
$$

It is easy to check that $p^{(m)}\left(i_{j}\right)=0$ for all $1 \leq j \leq n, 0 \leq m \leq r_{j}$. Evaluating at these points, we see that $r^{(m)}\left(i_{j}\right)=f^{(m)}\left(i_{j}\right)=a_{j}^{(m)}$. The uniqueness of the generalized Hermite interpolation polynomial, $H(x)$, shows then that $H(x) \equiv r(x) \in D[x]$.

Conversely, if the Hermite interpolation polynomial, $H(x)$, is in $D[x]$, it is indeed a polynomial generating the sequence $A$.

We proceed next to derive another characterization based on divided differences and Newton polynomials. Given a set of $n+\sum_{j=1}^{n} r_{j}$ points $\left(i_{j}, a_{j}^{(m)}\right) \in D^{2} \quad\left(1 \leq j \leq n, 0 \leq m \leq r_{j}\right)$ with distinct $i_{j}$. Recall, [5, p. 44], that the divided difference corresponding to these points is defined by

$$
[\underbrace{i_{1}, \ldots, i_{1}}_{r_{1}+1}, \underbrace{i_{2}, \ldots, i_{2}}_{r_{2}+1}, \ldots, \underbrace{i_{n}, \ldots, i_{n}}_{r_{n}+1}]=\sum_{j=1}^{n} \sum_{m=0}^{r_{j}} \frac{1}{m !} \frac{1}{\left(r_{j}-m\right) !} g_{j}^{\left(r_{j}-m\right)}\left(i_{j}\right) a_{j}^{(m)},
$$

where the functions $g_{j}$ 's are as defined in (3). Apart from the explicit shape in (2) (Hermite form), the unique interpolation polynomial corresponding to these points has another representation, 
known as its Newton form,

$$
\begin{aligned}
N(x)= & {\left[i_{1}\right]+\left[i_{1}, i_{1}\right] p_{1}(x)+\cdots+[\underbrace{i_{1}, \ldots, i_{1}}_{r_{1}+1}, i_{2}] p_{1}^{r_{1}+1}(x) } \\
& +[\underbrace{i_{1}, \ldots, i_{1}}_{r_{1}+1}, i_{2}, i_{2}] p_{2}(x)+\cdots+[\underbrace{i_{1}, \ldots, i_{1}}_{r_{1}+1}, \underbrace{i_{2}, \ldots, i_{2}}_{r_{2}+1}, i_{3}] p_{2}^{r_{2}+1}(x)+\cdots \\
& +[\underbrace{i_{1}, \ldots, i_{1}}_{r_{1}+1}, \underbrace{i_{2}, \ldots, i_{2}}_{r_{2}+1}, \ldots, \underbrace{i_{n}, \ldots, i_{n}}_{r_{n}+1}] p_{n}^{r_{n}}(x) \\
= & \sum_{j=1}^{n}\left(\sum_{q=1}^{r_{j}}[\underbrace{i_{1}, \ldots, i_{1}}_{r_{1}+1}, \ldots, \underbrace{i_{j}, \ldots, i_{j}}_{q+1}] p_{j}^{q}(x)+[\underbrace{i_{1}, \ldots, i_{1}}_{r_{1}+1}, \ldots, \underbrace{i_{j}, \ldots, i_{j}}_{r_{j}+1}, i_{j+1}] p^{r_{j}+1}(x)\right)
\end{aligned}
$$

where

$$
p_{j}^{q}(x)=\left(\prod_{h=1}^{j-1}\left(x-i_{h}\right)^{r_{h}+1}\right)\left(x-i_{j}\right)^{q} \quad\left(1 \leq j \leq n, 1 \leq q \leq r_{j}+1\right) .
$$

The elements $p_{0}(x):=1, p_{j}^{q}(x)$ are referred to as the Newton basis polynomials.

By the uniqueness of the interpolation polynomial and Theorem 2.1, we deduce that $A$ is a D-pol seq if and only if $N(x) \equiv H(x) \in D[x]$. Equating coefficients, we have:

Theorem 2.2. Keeping the above notation, the sequence $A$ is a D-pol seq (of order $R$ with respect to I) if and only if all the divided differences

$$
\left[i_{1}\right],[\underbrace{i_{1}, \ldots, i_{1}}_{r_{1}+1}, i_{2}], \ldots,[\underbrace{i_{1}, \ldots, i_{1}}_{r_{1}+1}, \underbrace{i_{2}, \ldots, i_{2}}_{r_{2}+1}, i_{3}], \ldots,[\underbrace{i_{1}, \ldots, i_{1}}_{r_{1}+1}, \ldots, \underbrace{i_{n}, \ldots, i_{n}}_{r_{n}+1}]
$$

are elements of $D$.

We collect now several special cases, whose straightforward verifications are omitted.

Corollary 2.2.1. Let $A=\left(a, a^{(1)}, \ldots, a^{(k)}\right) \in D^{k+1}$ and $c \in D$. Then

I. there exists $T(x)=b_{0}+b_{1}(x-c)+\cdots+b_{k}(x-c)^{k} \in D[x]$, where $b_{j}=a^{(j)} / j ! \in D_{Q}(j=$ $0,1,2, \ldots, k)$, such that $T^{(j)}(c)=a_{j}$ for all $j$.

II. A is a D-pol seq $\Longleftrightarrow b_{j} \in D$ for all $j \Longleftrightarrow j ! \mid a^{(j)}$ for all $j$.

III. $k ! A$ is a D-pol seq; moreover, $k$ ! is the least positive integer for which this is true for such sequence of length $k+1$.

\section{Difference polynomial sequences}

Throughout this section, we fix the sequence $I=(1,2, \ldots, n)$ and take $D=\mathbb{Z}$. Clearly, $\Delta^{k} \mathbb{Z}[x]_{n}$ is a subset of $\mathbb{Z}[x]_{n-k}$; it is indeed a proper subset as seen from the example $f(x)=3 x+1 \in \mathbb{Z}[x]_{2}$ which is not an element of $\Delta \mathbb{Z}[x]_{3}$ because $\Delta\left(a x^{2}+b x+c\right)=2 a x+a+b \neq 3 x+1$ when $a, b, c \in \mathbb{Z}$. However, it is easy to check that both $\Delta^{k} \mathbb{Z}[x]_{n}$ and $\Delta^{k} P_{n}$ are abelian groups under addition. In fact, they are isomorphic as we now show using the same technique as in [1]. 
Theorem 3.1. For $1 \leq k \leq n$, the group $\left(\Delta^{k} \mathbb{Z}[x]_{n},+\right)$ is isomorphic to $\left(\Delta^{k} P_{n},+\right)$.

Proof. Since for each $g(x) \in \Delta^{k} \mathbb{Z}[x]_{n}$, there is a polynomial $f(x) \in \mathbb{Z}[x]_{n}$ such that $\left(\Delta^{k} f\right)(x)=$ $g(x)$, define a map $v: \Delta^{k} \mathbb{Z}[x]_{n} \rightarrow \Delta^{k} P_{n}$ by

$$
v(g)\left(=v\left(\Delta^{k} f\right)\right):=\left(\Delta^{k} f(1), \Delta^{k} f(2), \ldots, \Delta^{k} f(n-k)\right) .
$$

It is easy to see that $v$ is an additive homomorphism. To show that $v$ is an isomorphism, it remains to show that $v$ is bijective. Let $\mathbf{c}=\left(c_{1}, c_{2}, \ldots, c_{n-k}\right) \in \Delta^{k} P_{n}$. Then there exists $f_{c}(x) \in \mathbb{Z}[x]$ such that

$$
\left(\Delta^{k} f_{c}\right)(i)=c_{i} \quad(1 \leq i \leq n-k) .
$$

Recall from (5) that the Newton basis polynomials of order $R=(0, \ldots, 0)$ corresponding to $I=(1,2, \ldots, n)$ are

$$
p_{0}(x):=1, p_{n}(x):=(x-1)(x-2) \cdots(x-n) \in \mathbb{Z}[x]_{n+1} \quad(n \in \mathbb{N}) .
$$

Since each $p_{n}(x)$ is monic, by the division algorithm,

$$
f_{c}(x)=q(x) p_{n}(x)+r(x),
$$

where $q, r \in \mathbb{Z}[x]$ with $\operatorname{deg} r \leq n-1$ or $r \equiv 0$. Let $m_{1}(x)=x q(x+1)-(x-n) q(x) \in \mathbb{Z}[x]$,

$$
m_{j}(x)=x m_{j-1}(x+1)-(x-n+j-1) m_{j-1}(x) \quad(2 \leq j \leq k) .
$$

Then

$$
\left(\Delta^{k} f_{c}\right)(x)=\left(\Delta^{k} q p_{n}\right)(x)+\left(\Delta^{k} r\right)(x)=p_{n-k}(x) m_{k}(x)+\left(\Delta^{k} r\right)(x),
$$

with $\operatorname{deg}\left(\Delta^{k} r\right) \leq n-k-1$, or $\Delta^{k} r \equiv 0$. Evaluating at $i \in\{1, \ldots, n-k\}$, we see that $\left(\Delta^{k} r\right)(x)$ generates the sequence $\mathbf{c}$, which shows that $v$ is surjective.

To show that $v$ is injective, let $g_{1}, g_{2} \in \Delta^{k} \mathbb{Z}[x]_{n}$ with $g_{1}=\Delta^{k} f_{1}, g_{2}=\Delta^{k} f_{2}\left(f_{1}, f_{2} \in \mathbb{Z}[x]_{n}\right)$ be such that

$$
\left(\Delta^{k} f_{1}(1), \ldots, \Delta^{k} f_{1}(n-k)\right)=v\left(g_{1}(x)\right)=v\left(g_{2}(x)\right)=\left(\Delta^{k} f_{2}(1), \ldots, \Delta^{k} f_{2}(n-k)\right),
$$

and so $\Delta^{k} f_{1}(i)=\Delta^{k} f_{2}(i)(1 \leq i \leq n-k)$. Since both $\operatorname{deg} \Delta^{k} f_{1}$ and $\operatorname{deg} \Delta^{k} f_{2}$ are $<n-k$, and the two polynomials agree at $n-k$ distinct points, they must be identical, i.e., $v$ is injective.

Our next result gives a necessary and sufficient condition for a sequence $\mathbf{c} \in \mathbb{Z}^{n-k}$ to be an element in $\Delta^{k} P_{n}$.

Theorem 3.2. Let $\mathbf{c}=\left(c_{1}, c_{2}, \ldots, c_{n-k}\right) \in \mathbb{Z}^{n-k}$ whose Newton interpolation polynomial (4) of order $R=(0, \ldots, 0)$ is

$$
N_{\mathbf{c}}(x)=\sum_{i=0}^{n-k-1} d_{i} p_{i}(x) \in \mathbb{Q}[x] .
$$

Then $\mathbf{c} \in \Delta^{k} P_{n}$ if and only if each $d_{i}$ is an integer divisible by

$$
(i+k) ! / i !=(i+1)(i+2) \cdots(i+k) \quad(0 \leq i \leq n-k-1) .
$$


Proof. If $\mathbf{c}=\left(c_{1}, c_{2}, \ldots, c_{n-k}\right) \in \mathbb{Z}^{n-k} \cap \Delta^{k} P_{n}$, then from the definition and the proof of Theorem 3.1 there is $f(x) \in \mathbb{Z}[x]_{n}$ satisfying

$$
\left(\Delta^{k} f\right)(i)=c_{i} \quad(1 \leq i \leq n-1)
$$

i.e., $\left(\Delta^{k} f\right)(x)$ generates c. The two polynomials $N_{\mathbf{c}}(x)$ and $(\Delta f)(x)$ being of degree $<n-k$ and agreeing on $n-k$ points, must be identical. Let the polynomial $f(x)$ be written with respect to Newton basis polynomials as

$$
f(x)=\sum_{i=0}^{n-1} b_{i} p_{i}(x)
$$

and so all coefficients $b_{i} \in \mathbb{Z}$. Using $\Delta^{k} p_{i}(x)=i(i-1) \cdots(i-k+1) p_{i-k}(x)$, we get

$$
\sum_{i=0}^{n-k-1} d_{i} p_{i}(x)=N_{\mathbf{c}}(x)=\left(\Delta^{k} f\right)(x)=\sum_{i=0}^{n-k-1}(i+1)(i+2) \cdots(i+k) b_{i+k} p_{i}(x) .
$$

Equating coefficients, we get

$$
d_{i}=(i+1)(i+2) \cdots(i+k) b_{i+k} \quad(0 \leq i \leq n-k-1)
$$

which shows that all $d_{i} \in \mathbb{Z}$ and $(i+1)(i+2) \cdots(i+k) \mid d_{i}$.

Conversely, if each coefficient $d_{i}$ in the Newton interpolation polynomial $N_{\mathbf{c}}(x)$ in (7) is an integer divisible by $(i+1)(i+2) \cdots(i+k)$, i.e., the relation (8) holds, then retreating the above steps, we see that the integers $b_{k}, \ldots, b_{n-1}$ are uniquely determined from the $d_{i}$ 's, while the integers $b_{0}, \ldots, b_{k-1}$ can be given arbitrarily. The sequence $\mathbf{c}$ is thus generated by $\left(\Delta^{k} f\right)(x)$ with $f(x)=\sum_{i=0}^{n-1} b_{i} p_{i}(x)$, showing that $c \in \Delta^{k} P_{n}$.

Remarks. In the last part of the proof of Theorem 3.2, the fact that the integer coefficients $b_{0}, \ldots, b_{k-1}$ can be chosen arbitrarily is a consequence of the fact that the operator $\Delta^{k}$ annihilates all polynomials of degree $\leq k-1$. Should these integers be required to be uniquely determined, one possible condition to be imposed is that $c \in \Delta^{j} P_{n}$ for all $j=1, \ldots, k$.

The algebraic structures of related quotient groups will be explicitly determined next.

Theorem 3.3. For positive integers $n \geq 2$ and $1 \leq k \leq n-1$, we have

$$
\begin{aligned}
& \text { I. } \mathbb{Z}^{n-k} / \Delta^{k} P_{n} \cong \mathbb{Z} / k ! \mathbb{Z} \oplus \mathbb{Z} /(k+1) ! \mathbb{Z} \oplus \mathbb{Z} /(k+2) ! \mathbb{Z} \oplus \mathbb{Z} /(k+3) ! \mathbb{Z} \oplus \cdots \oplus \mathbb{Z} /(n-1) ! \mathbb{Z} . \\
& \text { II. } P_{n-k} / \Delta^{k} P_{n} \cong \mathbb{Z} / \frac{k !}{0 !} \mathbb{Z} \oplus \mathbb{Z} / \frac{(k+1) !}{1 !} \mathbb{Z} \oplus \mathbb{Z} / \frac{(k+2) !}{2 !} \mathbb{Z} \oplus \cdots \oplus \mathbb{Z} / \frac{(n-1) !}{(n-k-1) !} \mathbb{Z} .
\end{aligned}
$$

Proof. I. Since the Newton polynomials $p_{i}(x) \quad(i=0,1, \ldots, n-1)$ as in (6), form a basis for the $\mathbb{Z}$-module $\mathbb{Z}[x]_{n}$, and for $j \geq k$ since

$$
\Delta^{k} p_{j}(x)=j(j-1) \cdots(j-k+1) p_{j-k}(x)=\frac{j !}{(j-k) !} p_{j-k}(x),
$$

the polynomials

$$
\frac{k !}{0 !} p_{0}(x), \frac{(k+1) !}{1 !} p_{1}(x), \frac{(k+2) !}{2 !} p_{2}(x), \ldots, \frac{(n-1) !}{(n-k-1) !} p_{n-k-1}(x)
$$


are elements of $\Delta^{k} \mathbb{Z}[x]_{n}$. In fact, it is easy to check that these elements form a $\mathbb{Z}$-basis for the $\mathbb{Z}$-module $\Delta^{k} \mathbb{Z}[x]_{n}$. The map $v: \Delta^{k} \mathbb{Z}[x]_{n} \rightarrow \Delta^{k} P_{n}$, as defined in Theorem 3.1, being an isomorphism shows then that the elements

$$
v\left(\frac{s !}{(s-k) !} p_{s-k}(x)\right) \quad(s=k, k+1, \ldots, n-1)
$$

form a $\mathbb{Z}$-basis for $\Delta^{k} P_{n}$. Let $C=\left(c_{i, j}\right)_{1 \leq i, j \leq n-k}$ be an $(n-k) \times(n-k)$ lower triangular matrix with

$$
\begin{aligned}
c_{i, j} & = \begin{cases}\{(k+j-1)(k+j-2) \cdots j\} \cdot\{(i-1)(i-2) \cdots(i-j+1)\} & \text { if } 2 \leq j \leq i \leq n-k \\
k ! & \text { if } j=1 \\
0 & \text { if } 1 \leq i<j \leq n-k .\end{cases} \\
& =(k+j-1) !\left(\begin{array}{c}
i-1 \\
j-1
\end{array}\right)
\end{aligned}
$$

Clearly, the elements

$$
v\left((k+j-1)(k+j-2) \cdots j p_{j-1}(x)\right)=v\left(\frac{(k+j-1) !}{(j-1) !} p_{j-1}\right) \quad(j=1,2, \ldots, n-k)
$$

represent the $j$ th column of $C$. Next, let $A=\left(a_{i, j}\right)_{1 \leq i, j \leq n-k}$ be an $(n-k) \times(n-k)$ lower triangular matrix with

$$
a_{i, j}=\left\{\begin{array}{ll}
\left(\begin{array}{l}
i-1 \\
j-1
\end{array}\right) & \text { if } 2 \leq j \leq i \leq n-k \\
1 & \text { if } j=1 \\
0 & \text { if } 1 \leq i<j \leq n-k,
\end{array}=\left(\begin{array}{l}
i-1 \\
j-1
\end{array}\right)\right.
$$

and denote the $j$ th column of $A$ by $e(j-1)(j \in\{1,2, \ldots, n-k\})$. Since $A$ is unimodular, i.e., $\operatorname{det} A=1$, we see that the elements $e(j-1)(j=1,2, \ldots, n-k)$ form a $\mathbb{Z}$-basis for $\mathbb{Z}^{n-k}$. Let $D$ be the $(n-k) \times(n-k)$ diagonal matrix whose $j^{\text {th }}$ diagonal entry is $(k+j-1)$ !, where $j=1,2, \ldots, n-k$. It is easily checked that $C=A D$, which in turn shows that

$$
v\left((k+j-1)(k+j-2) \cdots j p_{j-1}(x)\right)=(k+j-1) ! e(j-1) \quad(j=1,2, \ldots, n-k),
$$

and so $\{(k+j-1) ! e(j-1): j=1,2, \ldots, n-k\}$ forms a $\mathbb{Z}$-basis for $\Delta^{k} P_{n}$. Consequently, by $[3$, Chapters 6, 8]

$$
\begin{aligned}
\mathbb{Z}^{n-k} / \Delta^{k} P_{n} & =\frac{\langle e(0)\rangle \oplus\langle e(1)\rangle \oplus\langle e(2)\rangle \oplus \cdots \oplus\langle e(n-k-1)\rangle}{k !\langle e(0)\rangle \oplus(k+1) !\langle e(1)\rangle \oplus(k+2) !\langle e(2)\rangle \oplus \cdots \oplus(n-1) !\langle e(n-k-1)\rangle} \\
& =\frac{\langle e(0)\rangle}{k !\langle e(0)\rangle} \oplus \frac{\langle e(1)\rangle}{(k+1) !\langle e(1)\rangle} \oplus \frac{\langle e(2)\rangle}{(k+2) !\langle e(2)\rangle} \oplus \cdots \oplus \frac{\langle e(n-k-1)\rangle}{(n-1) !\langle e(n-k-1)\rangle} \\
& \cong \mathbb{Z} / k ! \mathbb{Z} \oplus \mathbb{Z} /(k+1) ! \mathbb{Z} \oplus \mathbb{Z} /(k+2) ! \mathbb{Z} \oplus \mathbb{Z} /(k+3) ! \mathbb{Z} \oplus \cdots \oplus \mathbb{Z} /(n-1) ! \mathbb{Z} .
\end{aligned}
$$

II. As seen above, the set $\{(j-1) ! e(j-1) \mid j=1, \ldots, n-k\}$ forms a $\mathbb{Z}$-basis for $P_{n-k}$, and from part I, the set $\{(k+j-1) ! e(j-1) \mid j=1,2, \ldots, n-k\}$ is a $\mathbb{Z}$-basis for $\Delta^{k} P_{n}$. Thus, by 
$[3$, Chapters 6,8$]$ we have

$$
\begin{aligned}
\frac{P_{n-k}}{\Delta^{k} P_{n}} & =\frac{0 !\langle e(0)\rangle \oplus 1 !\langle e(1)\rangle \oplus 2 !\langle e(2)\rangle \oplus \cdots \oplus(n-k-1) !\langle e(n-k-1)\rangle}{k !\langle e(0)\rangle \oplus(k+1) !\langle e(1)\rangle \oplus(k+2) !\langle e(2)\rangle \oplus \cdots \oplus(n-1) !\langle e(n-k-1)\rangle} \\
& =\frac{\langle e(0)\rangle}{k !\langle e(0)\rangle} \oplus \frac{\langle e(1)\rangle}{(k+1) !\langle e(1)\rangle} \oplus \frac{\langle e(2)\rangle}{3 \cdots(k+2)\langle e(2)\rangle} \oplus \cdots \oplus \frac{\langle e(n-2)\rangle}{(n-k) \cdots(n-1)\langle e(n-2)\rangle} \\
& \cong \mathbb{Z} / \frac{k !}{0 !} \mathbb{Z} \oplus \mathbb{Z} / \frac{(k+1) !}{1 !} \mathbb{Z} \oplus \mathbb{Z} / \frac{(k+2) !}{2 !} \mathbb{Z} \oplus \cdots \oplus \mathbb{Z} / \frac{(n-1) !}{(n-k-1) !} \mathbb{Z} .
\end{aligned}
$$

\section{Acknowledgements}

V. Kim acknowledges the support through the Royal scholarship under Her Royal Highness Princess Maha Chakri Sirindhorn Education Project to the Kingdom of Cambodia.

S. Prugsapitak wishes to thank the Algebra and Applications Research Unit, Department of Mathematics and Statistics, Faculty of Science, Prince of Songkla University.

\section{References}

[1] Cornelius Jr., E. F., \& Schultz, P. (2008). Sequences generated by polynomials, Amer. Math. Monthly, 115 (2), 154-158.

[2] Davis, P. J. (1975). Interpolation and Approximation, Dover, New York.

[3] Hartley, B., \& Hawkes, T. O. (1974). Rings, Modules and Linear Algebra, Chapman and Hall, London.

[4] Kim, V., Laohakosol, V. \& Prugsapitak, S. (2019). Sequences generated by polynomials over integral domains, Walailak J. Sci. Tech., 16 (9), 625-633.

[5] Spitzbart, A. (1960). A generalization of Hermite's interpolation formula, Amer. Math. Monthly, 67 (1), 42-46. 